\title{
Detection of cavitation in hydraulic turbines
}

\author{
Xavier Escaler ${ }^{\mathrm{a}, *}$, Eduard Egusquiza ${ }^{\mathrm{a}}$, Mohamed Farhat ${ }^{\mathrm{b}}$, \\ François Avellan $^{\mathrm{b}}$, Miguel Coussirat ${ }^{\mathrm{a}}$ \\ ${ }^{a}$ Centre de Diagnòstic Industrial i Fluidodinàmica (CDIF), Universitat Politècnica de Catalunya (UPC), \\ Avinguda Diagonal 647, 08028 Barcelona, Spain \\ ${ }^{\mathrm{b}}$ Laboratoire de Machines Hydrauliques (LMH-IMHEF), Ecole Polytechnique Fédérale de Lausanne (EPFL), \\ Avenue de Cour 33bis, CH-1007 Lausanne, Switzerland
}

Received 7 June 2004; received in revised form 10 August 2004; accepted 11 August 2004

\begin{abstract}
An experimental investigation has been carried out in order to evaluate the detection of cavitation in actual hydraulic turbines. The methodology is based on the analysis of structural vibrations, acoustic emissions and hydrodynamic pressures measured in the machine. The proposed techniques have been checked in real prototypes suffering from different types of cavitation. In particular, one Kaplan, two Francis and one Pump-Turbine have been investigated in the field. Additionally, one Francis located in a laboratory has also been tested.

First, a brief description of the general features of cavitation phenomenon is given as well as of the main types of cavitation occurring in hydraulic turbines. The work presented here is focused on the most important ones which are the leading edge cavitation due to its erosive power, the bubble cavitation because it affects the machine performance and the draft tube swirl that limits the operation stability.

Cavitation detection is based on the previous understanding of the cavity dynamics and its location inside the machine. This knowledge has been gained from flow visualisations and measurements in laboratory devices such as a high-speed cavitation tunnel and a reduced scale turbine test rig. The main techniques are the study of the high frequency spectral content of the signals and of their amplitude demodulation for a given frequency band. Moreover, low frequency spectral content can also be used in certain cases. The results obtained for the various types of cavitation found in the selected machines are presented and discussed in detail in the paper. Conclusions are drawn about the best sensor, measuring location, signal
\end{abstract}

\footnotetext{
*Corresponding author. Tel.: +34934012599; fax: + 34934015812.

E-mail address: escaler@mf.upc.es (X. Escaler).
} 
processing and analysis for each type of cavitation, which serve to validate and to improve the detection techniques.

(C) 2004 Elsevier Ltd. All rights reserved.

Keywords: Cavitation detection; Hydraulic turbines; Vibrations; Amplitude demodulation

\section{Introduction}

Cavitation in hydraulic machinery presents unwanted consequences such as flow instabilities, excessive vibrations, damage to material surfaces and degradation of machine performance. Unfortunately, these problems are becoming more important because the propensity for cavitation is being enhanced. In first place, the growth of the turbine output power is based on the reduction of dimensions to decrease the cost of its components. Hence, the speeds are being increased and the cavitation number is thereby decreased. In second place, there is a trend to operate the turbines in conditions far from their best efficiency point imposed by the deregulation of the hydropower generation market and cavitation phenomena are more prone to occur at offdesign operating conditions. Therefore, the combination of both factors is certainly promoting the risk of cavitation problems in hydraulic machines. Since corrective procedures are very difficult to apply in existing units, monitoring systems for cavitation detection during operation that serve to avoid harmful situations seem to be the best solution.

In this sense, although cavitation within pumps has been the subject of extensive research up to now, as demonstrated by the works from Coutier-Delgosha et al. [1], Cudina [2], Baldassarre et al. [3], McNulty and Pearsall [4] and others, it must be noted that few studies have been published related to cavitation within hydropower turbines. Because of that, the current paper is mainly focused in detection of cavitation in hydro turbines.

The cavitation detection methods under study in this work are based on induced vibrations and pressures measured in the machine. This approach is advantageous since hydropower generation is not affected. Such detection methodologies have been developed from basic knowledge of the cavitation phenomena reproduced in laboratory under simple and controlled conditions, see for instance the work of Avellan and Henry [5] and Bourdon et al. [6]. Nevertheless, when they have to be used in actual prototypes their correct application becomes more complex because cavitation can take different forms and appear on various locations depending on the machine operating condition. In fact, the global dynamic behaviour of the machine, other possible sources of excitation and the transmission paths must also be taken into account to interpret the signals. So, a brief description of the basic cavitation phenomena and the different forms that can take place in hydraulic turbines is given first. This information is completed with an explanation of the type of measurements and the signal processing techniques used for detection in the field. Then, the experimental results are presented and discussed.

\section{General features of cavitation}

\subsection{Definition}

Cavitation is defined by Knapp et al. [7] as the condition when a liquid reaches a state at which vapour cavities are formed and grow due to dynamic-pressure reductions to the vapour pressure 
of the liquid at constant temperature. In a flowing liquid, these cavities are subjected to a pressure increase that stops and reverses their growth, collapsing implosively and disappearing. The violent process of cavity collapse takes place in a very short time of about several nanoseconds and results in the emission of large amplitude shock-waves, as demonstrated by Avellan and Farhat [8]. A high-speed reentrant liquid micro-jet directed towards the boundary can also occur for cavities collapsing close to a solid surface [9]. If the amplitude of the resulting pressure pulse is larger than the limit of the material mechanical strength, a hollow or indentation of several micrometers called "pit" will be formed on the surface. If an accumulation of pits takes place in a narrow area, the material is finally eroded and mass loss occurs due to the repetitive action of the cavity collapses. In a flowing liquid, these cavities can take different forms that can be described as travelling bubbles, attached cavities or cavitating vortices. For general information on the subject refer to the reviews of Hammit [10] and Arndt [11].

\subsection{Travelling bubbles}

Bubbles usually appear around a body from micron-sized nuclei in low pressure regions of the flow (see left of Fig. 1). Travelling with the flow, they implode when they find an adverse pressure gradient. These bubbles are strongly influenced by the air content of the liquid. Nevertheless, their erosive power is considered to be relatively weak. An isolated bubble can be modelled based on the assumption that remains spherical in an infinite liquid [12]. In this case, the generalised Rayleigh-Plesset equation is a valid approximation of the bubble growth and it can be solved to find the radius of the bubble, $R_{B}(t)$, provided that the bubble pressure, $p_{B}(t)$, and the infinite domain pressure, $p_{\infty}(t)$, are known:

$$
\frac{p_{B}(t)-p_{\infty}(t)}{\rho}=R_{B} \frac{\mathrm{d}^{2} R_{B}}{\mathrm{~d} t^{2}}+\frac{3}{2}\left(\frac{\mathrm{d} R_{B}}{\mathrm{~d} t}\right)^{2}+\frac{4 v}{R_{B}} \frac{\mathrm{d} R_{B}}{\mathrm{~d} t}+\frac{2 \gamma}{\rho R_{B}} .
$$

Assuming that the bubble reaches a maximum radius, $R_{0}$, from which the implosion or collapsing process starts, the Rayleigh time or collapse time, $\tau$, until $R_{B}=0$ is reached is given by

$$
\tau=0.915 R_{0} \sqrt{\frac{\rho}{p_{\infty}-p_{v}}} .
$$

The theoretical work of Rayleigh on an imploding spherical cavity also permits to calculate the emission process of high pressure pulses by the collapsing bubbles when their radii tend towards
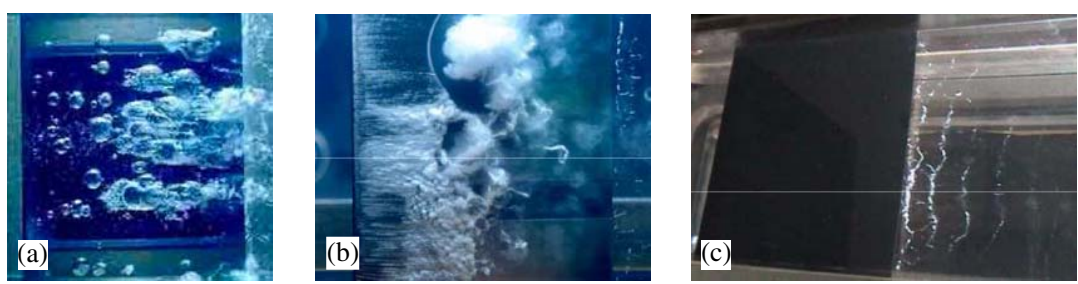

Fig. 1. Types of cavitation on a 2D hydrofoil (flow from left to right): (a) travelling bubbles, (b) unstable attached sheet and (c) Von Karman vortex-shedding. 
zero which represents the potential for the generation of shock waves and noise. However, this formulation only gives approximate results because the viscosity and surface tension effects have been neglected and it is supposed that the bubble only contains vapour. Other limiting assumptions are to consider that the bubbles remain spherical and that they are isolated, which is often far from the reality in cavitating flows, especially in the vicinity of a boundary. So, experimental work is needed to characterise the dynamics and acoustics of bubbles interacting with each other in real flows.

\subsection{Attached cavities}

Cavitation can take the form of macro-cavities that develop an attach on a solid wall placed in the flow. For instance, the so-called partial cavitation grows from the leading edge on the suction side of a hydrofoil with a positive angle of incidence. This is a very common and complex type of cavitation that can present different regimes depending on the hydrodynamic conditions.

One of the regimes, sheet cavitation, is characterised by thin stable cavities with smooth and transparent interfaces. At their rear part, the cavity closure presents a slight and weak pulsation due to the shedding of small cavitation vortices so that it represents a low risk of erosion.

Another regime, cloud cavitation, shows a strong unsteadiness and a pulsating behaviour that provokes significant oscillations of the cavity length (see centre of Fig. 1). The cavity interface is wavy and turbulent. Large U-shaped transient cavities and clouds of cavities are shed away downstream of the cavity closure that collapse violently on the solid surface (see the work of Avellan et al. [13]). Consequently, this is a very aggressive form of cavitation with a high erosive power. When this type of cavitation occurs in turbomachinery it can induce abnormal dynamic behaviour and cause serious erosion.

\subsection{Vortex cavitation}

Flow regions with concentrated vorticity can develop cavitation in their central cores due to the low pressures generated. If the tips of these vapour filled vortices are in contact with a solid surface they become potentially erosive since the final collapse of the whole cavity takes place on them. A typical example of this type of cavitation can develop if Von Karman vortex-shedding occurs at the trailing edge of a hydrofoil and pressure is low enough (see right of Fig. 1). As a result, lift fluctuations are provoked synchronised with the shedding frequency. Vortex cavitation can also occur in the flow channels of hydraulic machines when they operate at part load. In the case of a coupling phenomena with a natural frequency this can lead to fatigue damage.

\section{Cavitation in hydraulic turbines}

\subsection{Turbine type and configuration}

Cavitation plays an important role in reaction water turbines such as Kaplan, Francis and Pump-Turbine. The main difference between Kaplan and Francis turbines is the design of the runner which is, respectively, axial and radial. For the reversible Pump-Turbine, the runner has a 
radial design with low specific speed and it can operate in turbine or in pump mode. The rest of the machine components comprising the penstock, spiral casing, stay vanes, guide vanes, draft tube, shaft, alternator and bearings are analogous for any particular design. In Fig. 2, a schematic of a Kaplan turbine is shown on the left and a cross section of a Francis runner with its downstream reservoir is shown on the right. The runner design has a clear influence on the cavitation phenomenon but two other important parameters also influence its inception and development which are the machine setting level and the operation at off-design conditions.

\subsection{Turbine setting level}

The setting level of the machine is the distance $H_{s}$ indicated in Fig. 2 that determines the pressure field in relation to the vapour pressure threshold. For instance, bubble cavitation can appear even at the best efficiency point of the machine because it has a strong dependence on this level. Thus, the cavitation coefficient of a hydraulic turbine depends on this parameter (for more information refer to Franc et al. [14]). The International Electrotechnical Commission (ICE) recommends to use the Thoma number or plant cavitation number $\sigma_{p}$ defined as

$$
\sigma_{p}=\frac{N P S E}{E},
$$

where $E$ is the specific energy and NPSE is the net positive suction specific energy that in turn can also be calculated as

$$
N P S E=\frac{p_{I}}{\rho}+g\left(Z_{I}-Z_{\mathrm{ref}}\right)+\frac{1}{2} C_{I}^{2}-\frac{p_{v}}{\rho}
$$

or

$$
N P S E=\frac{p_{a}}{\rho}-g H_{S}+\frac{1}{2} C_{I}^{2}-\frac{p_{v}}{\rho} .
$$
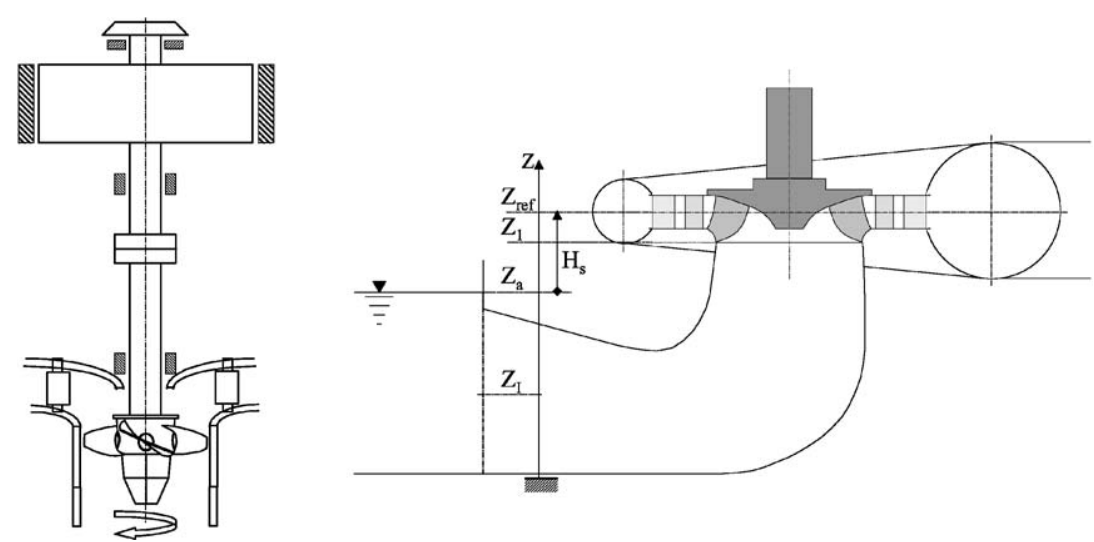

Fig. 2. (Left) Schematic of a Kaplan turbine and its mechanical configuration. (Right) Schematic of a Francis runner, draft tube and downstream reservoir. 


\subsection{Turbine operation}

A water turbine is designed to have the maximum efficiency for a given head and flow rate, but it can also operate at off-design points. As an example, the typical diagram for a Francis turbine is shown on the left of Fig. 3. The operating point is determined by the pressure or head coefficient, $\psi$, defined as

$$
\psi=\frac{2 E}{\omega^{2} R^{2}}
$$

and by the flow coefficient, $\varphi$, defined as

$$
\varphi=\frac{Q}{\pi \omega R^{3}} .
$$

The sub-index ${ }_{\Lambda}$ denotes the best efficiency or design point. Nevertheless, the head can change because the upstream or downstream reservoir levels may vary. On the other hand, the flow rate is changed by opening or closing the guide vane angle, $\beta$, in order to regulate the power output (see the schematic on the right of Fig. 3). These variations have a direct influence on the kinematics of the flow trough the runner that in turn determines the tendency to promote cavitations. The absolute fluid velocity in a fixed frame of reference is denoted by $C$ and the relative velocity in a rotating frame of reference is denoted by $W$ (subscripts 1 and 2 designate particular values at runner inlet and outlet). They are related by $U$ which is the tangential velocity. Then, the angle between $W$ and the tangent to the blade at the leading edge is the incidence angle, $\alpha$, of the incoming flow. If, for instance, there is an increase of head for a given guide vane angle, $\beta$, the absolute fluid velocity $C_{1}$ will grow and the incidence angle, $\alpha$, that for the design condition is very small and close to zero, will become positive. Consequently, when the machine operates at higher heads than design ones $\left(\psi / \psi_{\Lambda} \gg 1\right)$, attached cavitation can appear in the suction sides of
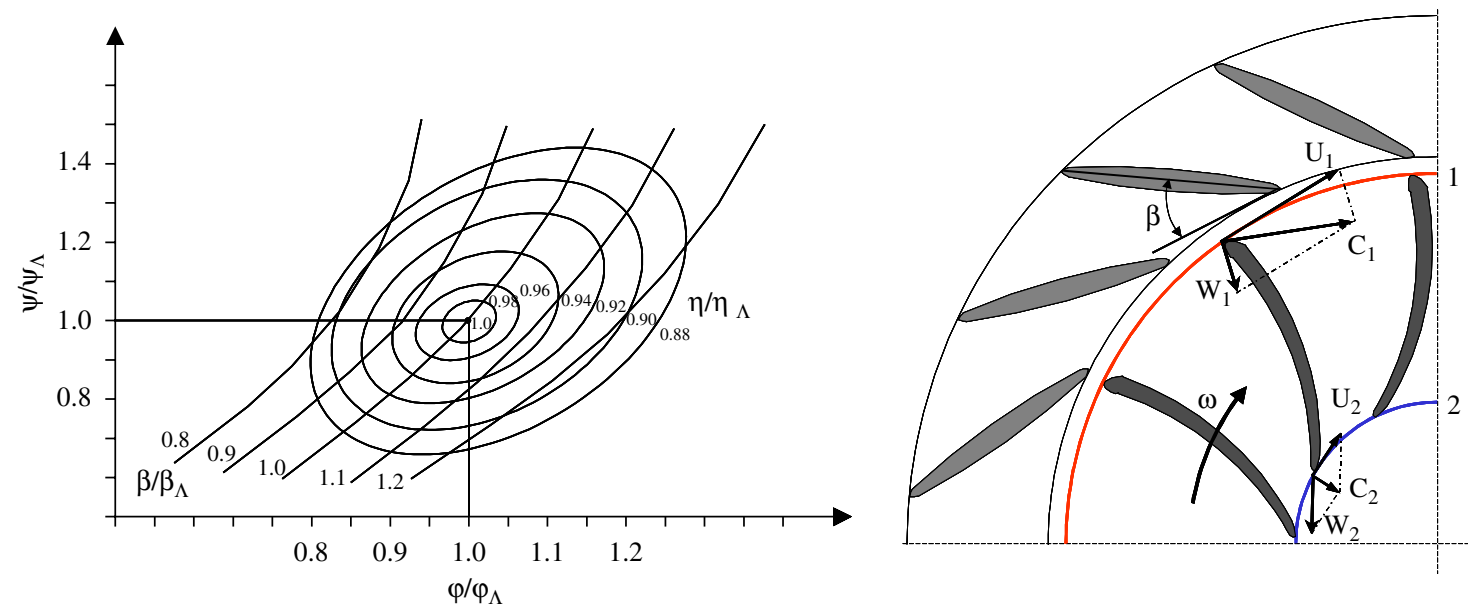

Fig. 3. (Left) Typical operating range for a Francis turbine. (Right) Vector diagrams describing the kinematics of the flow through a Francis turbine runner. 
the blades. Obviously, the velocity vectors at the outlet are also modified if the inlet conditions change. For example, the absolute velocity $C_{2}$, which has a radial direction at the best efficiency point, can change and leave a residual circumferential component affecting the draft tube flow. Readers requiring an overall vision of hydraulic turbines and power generation please refer to Creager and Justin [15], Raabe [16] and Vivier [17].

\subsection{Main types of cavitation}

The main forms of cavitation that can arise on Francis turbines are briefly described in the following paragraphs. More details can be found in Li [18].

\subsubsection{Leading edge cavitation}

See (1) in Figs. 4 and 5. It takes the form of an attached cavity on the suction side of the runner blades due to operation at a higher head than the machine design head $\left(\psi / \psi_{\Lambda}>1\right)$ when the incidence angle of the inlet flow is positive and largely deviated from the design value $(\alpha>0)$. It can also occur on the pressure side during operation at a lower head than the machine design head $\left(\psi / \psi_{\Lambda}<1\right)$ when the incidence angle is negative $(\alpha<0)$. If unstable, this is a very aggressive type of cavitation that is likely to deeply erode the blades and to provoke pressure fluctuations.

\subsubsection{Travelling bubble cavitation}

See (2) in Figs. 4 and 5. It takes the form of separated bubbles attached to the blade suction side near the mid-chord next to the trailing edge. These travelling bubbles appear due to a low plant cavitation number $\sigma_{p}$ and they grow with load reaching their maximum when the machine operates in overload condition with the highest flow rate $\left(\varphi / \varphi_{\Lambda} \gg 1\right)$. This is a severe and noisy type of cavitation that reduces significantly the machine efficiency and that can provoke erosion if the bubbles collapse on the blade.

\subsubsection{Draft tube swirl}

See (3) in Figs. 4 and 5. It is a cavitation vortex-core flow that is formed just below the runner cone in the centre of the draft tube. Its volume depends on $\sigma_{p}$ and it appears at partial load $\left(\varphi / \varphi_{\Lambda}<1\right)$ and at overload $\left(\varphi / \varphi_{\Lambda}>1\right)$ due to the residual circumferential velocity component of the flow discharged from the runner. This vortex rotates in the same direction as the runner at part load and in the opposite direction at overload. From $50 \%$ up to $80 \%$ of the best efficiency flow rate, the vortex core takes a helical shape and presents a precession rotation at $0.25-0.35$ times the runner rotating speed. In this case, circumferential pressure pulsations are generated at this low frequency. Strong fluctuations may occur if the precession frequency matches one of the free natural oscillation frequencies of the draft tube or penstock. This provokes large bursts of pressure pulses in the draft tube causing strong vibrations on the turbine and even on the powerhouse. Beyond the best efficiency point the vortex is axially centred in the draft tube cone.

\subsubsection{Inter-blade vortex cavitation}

See (4) in Figs. 4 and 5. This is formed by secondary vortices located in the channels between blades that arise due to the flow separation provoked by the incidence variation from the hub to the band. They can attach to the intersection of the blade inlet-edge with the crown or mid-way of 

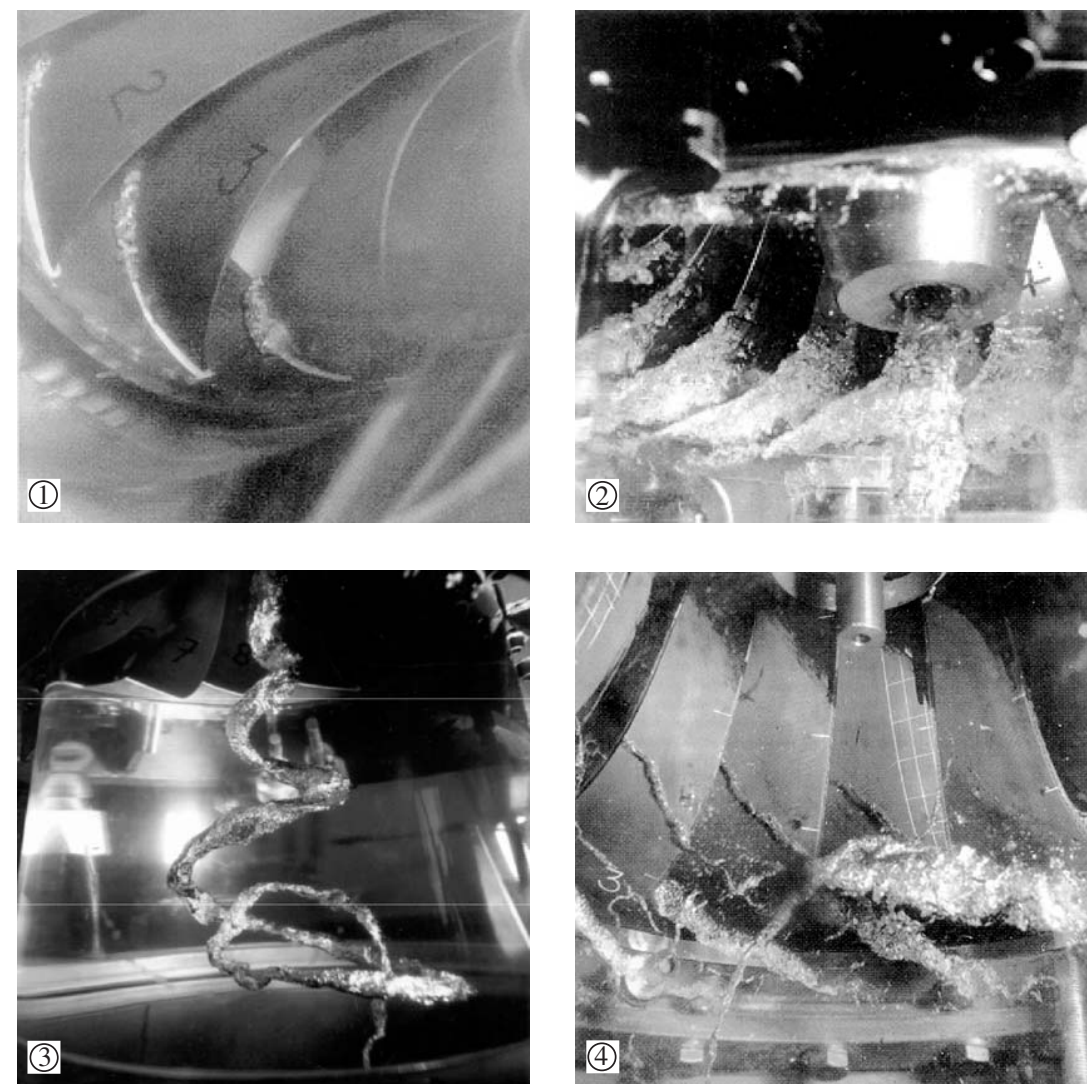

Fig. 4. Main types of cavitation in Francis turbines: (1) leading edge cavitation, (2) travelling bubble cavitation, (3) draft tube swirl and (4) inter-blade vortex cavitation. Pictures 1 and 4 courtesy of Franc et al. [14]. Picture 2 courtesy of Grindoz [19].
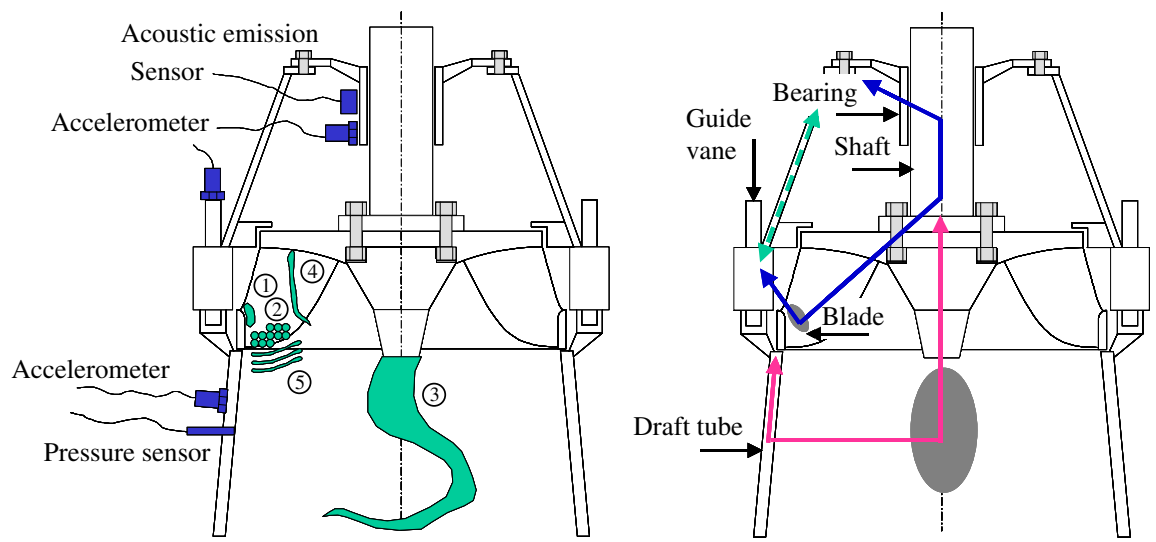

Fig. 5. Schematic of a Francis turbine runner showing: (left) various types of cavitation and location of transducers; (right) possible transmission paths followed by excitations acting on the blades and inside the draft tube just below the runner cone. 
the crown between the blades close to the suction side. Only if their tip is in touch with the runner surface they can result in erosion. These vortices appear at partial load operation $\left(\varphi / \varphi_{\Lambda}<1\right)$ and yield a high broadband noise level. They can also appear and cavitate at extremely high-head operation ranges $\left(\psi / \psi_{\Lambda} \gg 1\right)$ because the $\sigma_{p}$ is relatively low. In this case, they become unstable and cause strong vibrations.

\subsubsection{Von Karman vortex cavitation}

See 5 in Fig. 5. From the trailing edge of blades and vanes periodic vortex-shedding can occur. Severe pulsations and singing noise can be caused if a lock-in phenomenon occurs. As a result, the trailing edge might be damaged.

For Kaplan turbines, leading edge cavitation is less likely to occur than in Francis turbines. This is because the runner blades have a variable pitch and the machine always operates in "on cam" condition. This means that $\alpha$ is close to the optimum value over a wide operating range thanks to the good combination between runner blade inlet angle and guide vane angle. For the same reason, draft tube swirl is also less severe than in Francis turbines. Travelling bubble cavitation can take place too on the blades suction side due to heavy blade loading. An exclusive type of cavitation of Kaplan turbines is tip vortex cavitation. This cavitation arises in the gap or clearance between the blade tip and the casing. It is a strong type of cavitation that damages an area along the mid-chord length on the periphery of the blade suction side because the vortex tip touches the blade surface. The area on the blade tip end can also suffer erosion.

\subsection{Features of the excitation}

Cavitation is an unsteady phenomenon that provokes low frequency pressure oscillations and high frequency pressure pulses. The pressure oscillations are associated to the cavity dynamics and the pressure pulses are produced by the cavity collapses. As a result, vibrations and acoustic noise are generated and propagated trough the hydrodynamic and mechanical systems. Therefore, cavitation is a source of excitation that acts inside the main flow or adjacent to a solid wall. Hence, accelerometers and acoustic emission sensors, mounted on the external walls of the fixed components, and dynamic-pressure transducers, flush-mounted to the wetted walls, are useful for detection. For example, some typical measuring positions are indicated in Fig. 5.

\section{Detection techniques}

The methods to detect cavitation in real machines are based on the measurement and the analysis of the induced signals. Detection is not an easy task because, depending on the turbine design and the operating condition, the type of cavitation, its behaviour and its location are different. So, this affects the nature of the excitation and determines the transmission path followed up to the sensor. Furthermore, the measured signals can be contaminated by noise coming from other excitation sources of hydrodynamic, mechanical or electromagnetic origin. Therefore, the selection of the most adequate sensor and measuring position on the machine is of relevant importance to improve the detection as pointed out by Causon [20], Steller and Kirejczyk 
[21], Abbot et al. [22], Bajic and Keller [23] and Bourdon et al. [24]. For vibration measurements, common locations are the turbine guide bearing pedestal, the guide vane arm and the draft tube wall, meanwhile for pressure measurements the possible places are the draft tube and the spiral casing walls. In addition, measurements have to be carried out at different operating conditions to monitor the complete machine operating range. Finally, the measured signals must be recorded with a sufficiently high sampling frequency.

\subsection{Structure and fluid-borne noise}

The measurement and analysis of hydraulic noise are useful for investigating the features of the cavitation phenomenon as demonstrated by Baiter [25] and Ceccio and Brennen [26]. For real machines, the pioneer work of Abbot [27] is an example of how this correlation can be used in industrial applications such as hydro turbines to monitor erosive cavitation. In fact, structureborne noise can be easily measured in turbines. Fluid-borne noise can also be used but sometimes the location of a pressure sensor close to the runner is not possible. Anyway, it must be taken into account that the cavitation noise cannot be directly measured because the signals are attenuated during their propagation. In spite of that, the spectral content of the high frequency signals and the modulating frequencies still can be used for detection.

\subsubsection{High frequency content}

The study of vibration, acoustic emission and dynamic-pressure levels in the high frequency range is a well-known technique to detect cavitation activity (see [28]). The amplitude of a given frequency band can be compared for the various operating conditions by computing the autopower spectrum of the time signals. A uniform and sharp increase of this band in comparison with a cavitation-free situation can indicate the presence of cavitation. The use of acoustic emission sensors serves to extend this analysis to upper frequencies that the accelerometers cannot reach. The information given by the high frequency spectral content sometimes is not conclusive because other excitations such as rubbing can also provoke this symptom. So, an amplitude demodulation technique has to be used to improve the diagnostic.

\subsubsection{Amplitude demodulation}

The detection of the distinctive hydrodynamic frequencies governing the cavity dynamics can be used for cavitation identification. In a 2D hydrofoil tested in a cavitation tunnel, the dynamic behaviour of an unstable attached cavity is governed by a Strouhal law as proved from flow visualisations. This frequency can be detected applying amplitude demodulation techniques to high frequency pressure and vibration measurements as demonstrated by Farhat et al. [29], Abbot et al. [30] and Callenaere et al. [31]. In a real machine, it is believed that a modulating frequency should also exist and that it should be related to the natural oscillation of a biphasic flow or should be forced by the interaction phenomena and/or the flow instabilities. An example of this approach can be found in the work of Kaye and Farhat [32] for a model Francis turbine. This work intends to classify different types of cavitation using vibration analysis, but unfortunately the approach has not been extended to the real prototypes.

The demodulation procedure has to start with the filtering of the time domain signals in a wide frequency band of about several $\mathrm{kHz}$ to remove low frequency content. Then the amplitude 
envelope of the filtered signal is computed using an algorithm based on the Hilbert transform. Finally, the averaged auto-power spectrum of several analytic signals is obtained with a high resolution. Mathematically, the Hilbert transform, $H i$, of a time signal, $x(t)$, is defined as

$$
H i\{x(t)\}=\frac{1}{\pi} \int_{-\infty}^{\infty} x(\tau) \frac{1}{t-\tau} \mathrm{d} \tau .
$$

And the envelope is obtained by forming the analytical signal, $\stackrel{\nabla}{x}(t)$, that is a complex time signal whose imaginary part is the Hilbert transform of the real part. Thus, if

$$
\tilde{x}(t)=H i\{x(t)\},
$$

then

$$
\stackrel{\nabla}{x}(t)=x(t)+j \tilde{x}(t) .
$$

The analysis of the resulting envelope in the frequency domain permits to identify frequency values associated to the dynamic behaviour of the cavities. For instance, the occurrence of cavitation can be identified when a frequency related to some machine hydrodynamic phenomenon modulates the signals. The operating regimes with maximum cavitation severity are indicated by the highest amplitude levels of this peak. If necessary, this technique can be improved with the use of synchronous time averaged signals in order to amplify the cavitation excitation in front of other sources of noise that are not periodic. This consists in reducing to the mean the equivalent time signals starting at exactly the same shaft position. For example, Vizmanos et al. [33] have applied it to obtain better demodulation results while avoiding the need of large data storage in the computing system.

\subsection{Low frequency content}

Cavitation vortices and unstable cavities with a large oscillating volume provoke the perturbation of the main flow and result in strong pressure pulsations inside the hydraulic system. This is the case of the draft tube swirl in certain flow conditions. This low frequency fluctuation can be detected by the use of pressure transducers flush-mounted on the draft tube wall. If the intensity of the fluctuation is strong, the detection can also be made from structural vibrations. So, in this case, the procedure only requires the analysis of the frequency content of the pressure and vibration signals within a low frequency range.

\section{Experimental investigation}

From a practical point of view the most important types of cavitation affecting the operation of the water turbines are the inlet leading edge cavitation due to its erosive capability, the bubble cavitation at the outlet of the blades because it affects the performance and efficiency of the machine and the draft tube swirl that limits the stability of the operation. 


\subsection{Test cases}

These different types of cavitation have been investigated in four prototypes and in a reduced scale model. Table 1 summarises the type of machine and the type of cavitation. Moreover, the machine rotating speed, $N$, the number of runner blades, $Z_{b}$, and the number of guide vanes, $Z_{v}$, are also indicated.

To designate the measuring positions a combination of letters and numbers is used. The letter indicates the type of sensor, being A for accelerometer, AE for acoustic emission sensor and $\mathrm{P}$ for pressure sensor. In the case of accelerometers, if they are located on a guide bearing no additional letters are used but, for guide vane and draft tube measurements, GV and DT are added, respectively. Numbers are used to define the angular location referred to the penstock as indicated in Fig. 6. For sensors mounted in radial direction the numbers go from 11 to 14 and for sensors mounted in axial direction the numbers go from 15 to 18, always starting parallel to the penstock and advancing $90^{\circ}$ in the sense of the turbine rotation.

\subsection{Instrumentation}

A complete measurement set-up has been used for field measurements. Vibrations have been measured with general purpose piezoelectric accelerometers with mounted natural frequencies of about $40 \mathrm{kHz}$ and with acoustic emission sensors having a resonance frequency of about $200 \mathrm{kHz}$. The vibration sensors have been calibrated prior to the measurements to verify their sensitivity and typical frequency response. Dynamic pressures have been measured with piezoelectric pressure sensors. A photoelectric tachometer probe has been used for contact-free detection of the shaft rotation. The transducers have been conditioned and filtered to obtain the signals that have been recorded with a RACAL V-Store tape recorder. A frequency band per channel of $100 \mathrm{kHz}$ has been used. Once in the laboratory, the signals have been replayed and digitised using a 12-bit $1-\mathrm{MHz}$ PC data acquisition system. For some investigations a SONY Digital recorder PC208AX has also been used. For the tests in the laboratory model a LeCroy 6810 acquisition system has been used with a sampling frequency of $50 \mathrm{kHz}$ per channel.

\section{Detection of leading edge cavitation}

Unstable leading edge cavitation has to be detected to avoid runner damage. In a 2D hydrofoil in a water tunnel, its dynamic behaviour is governed by a Strouhal law, but this is not observed in

Table 1

Description of the turbines and the type of cavitation investigated

\begin{tabular}{|c|c|c|c|c|c|}
\hline Size of machine & Type of turbine & Type of cavitation & $N(\mathrm{rpm})$ & $Z_{b}(-)$ & $Z_{v}(-)$ \\
\hline \multirow[t]{4}{*}{ Prototype } & Kaplan & Leading edge & 125 & 6 & 24 \\
\hline & Francis 1 & Leading edge & 250 & 15 & 24 \\
\hline & Francis 2 & Draft tube swirl & 250 & 15 & 24 \\
\hline & Pump-Turbine & Draft tube swirl & 600 & 7 & 16 \\
\hline Model & Francis & Bubble & 974 & 19 & 20 \\
\hline
\end{tabular}




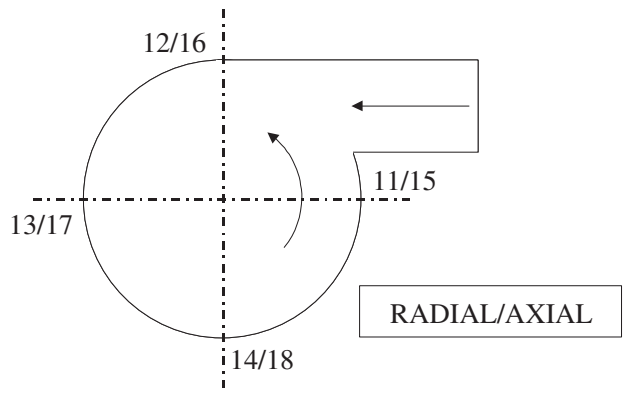

Fig. 6. Outline of a turbine transversal section with the reference figures used to define the measuring locations in radial and axial directions.

prototypes. Other frequencies appear to drive the shedding process of erosive cavities which are mainly the blade passing frequency, $f_{b}$, and the guide vane passing frequency, $f_{v}$, as indicated by the works of Abbot [27], Bourdon et al. [34], Farhat et al. [35,36] and Escaler et al. [37]. As a result, a modulated mechanical excitation acts on the blades due to collapsing cavities. The resulting high frequency vibration is transmitted through the rotating shaft to the guide bearing pedestal. Besides, hydrodynamic noise is generated at the same time by the shock waves and it can propagate through the liquid to the guide vanes and reach the bearing pedestal following a different path as indicated on the right of Fig. 5. Therefore, the best measuring locations are the closest to the runner but, depending on the location of the remote sensor and the transmissibility properties of the path from the excitation source, the characteristics of the induced signals can change in terms of amplitude and frequency content. To check this it is necessary to compare measurements in various locations. As follows, results are presented for a Kaplan and for a Francis.

\subsection{Kaplan turbine prototype}

This research work has been carried out in a vertical Kaplan turbine suffering erosive inlet cavitation on the blades. Photographs of the erosion appeared on one of the blades are shown in Fig. 7. This turbine has a nominal flow rate of $225 \mathrm{~m}^{3} / \mathrm{s}$ and a net head of $34 \mathrm{~m}$. The maximum output power is about $73 \mathrm{MW}$. The rotating speed is $125 \mathrm{rpm}\left(f_{f}=2.08 \mathrm{~Hz}\right)$. It has six blades and 24 guide vanes. The rotor is assembled to the foundation through two guide sleeve bearings, one above the runner and the other below the alternator. In between them, there is a thrust bearing.

Vibrations have been measured on the turbine guide bearing pedestal with one accelerometer mounted in radial position A13 and another one at $90^{\circ}$. On the thrust bearing, two radial accelerometers have also been mounted in similar positions together with a third one in axial direction. The turbine has been operated from minimum to maximum load at 10, 22, 32, 43 and $56 \mathrm{MW}$.

Comparing the vibration levels at various operating conditions, the amplitude of the higher frequencies increases with output power as the machine goes from 22 to $56 \mathrm{MW}$. The sharpest increase occurs at the maximum output power of $56 \mathrm{MW}$. In all the measuring positions, broadband vibration is detected above $3 \mathrm{kHz}$ specially at this maximum condition. As an example, the averaged power spectral density $(P S D)$ up to $10 \mathrm{kHz}$ of vibrations measured on position A13 


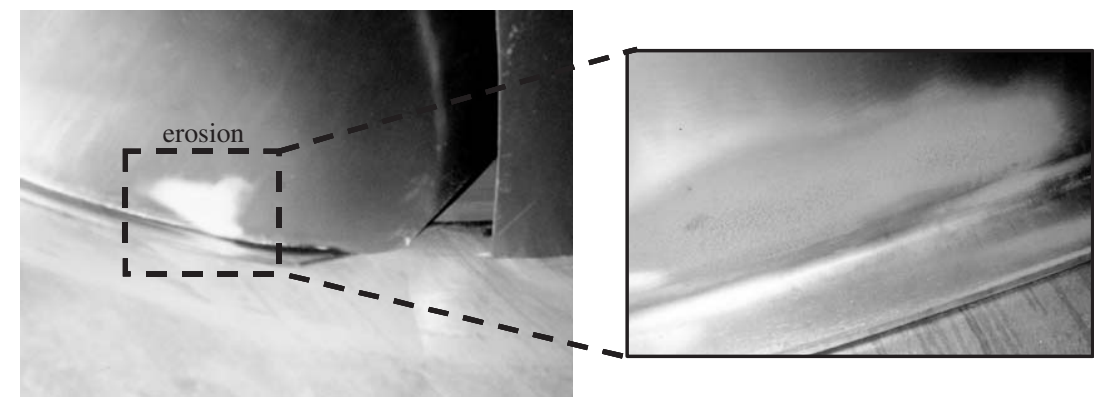

Fig. 7. Photographs of cavitation erosion on the runner blades of the Kaplan prototype.
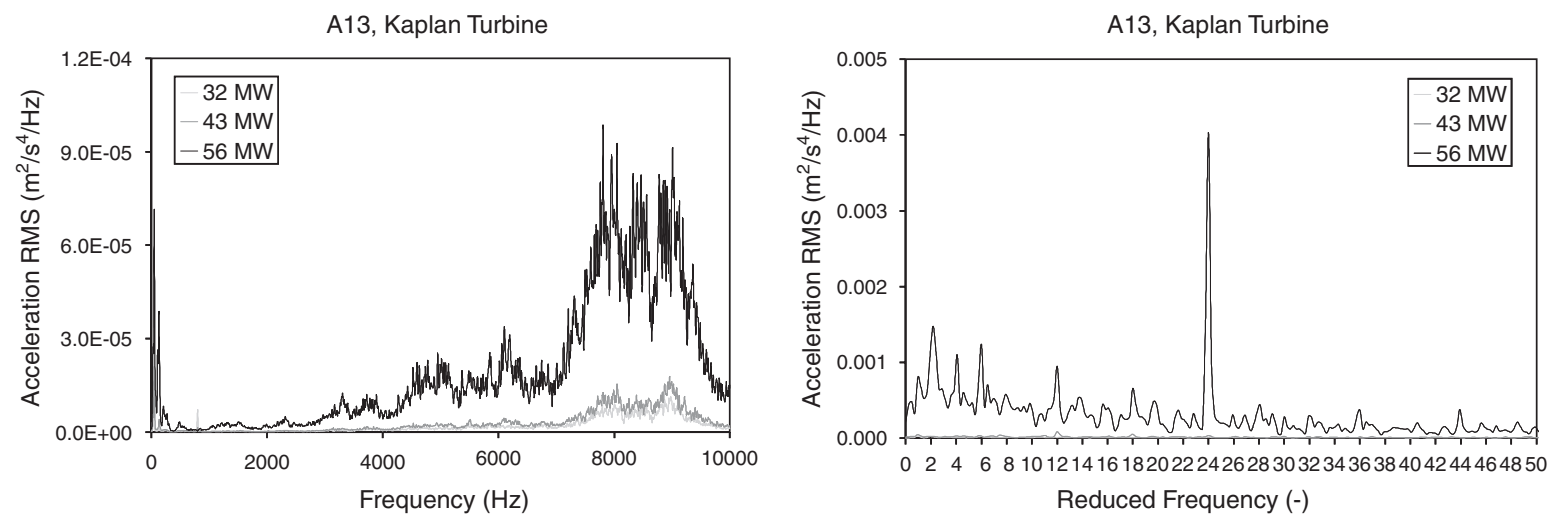

Fig. 8. Vibrations in A13 as a function of operating condition for the Kaplan turbine prototype. (Left) $P S D$ of raw spectra. (Right) PSD of modulation spectra in the band from 5 to $10 \mathrm{kHz}$.

are shown for 32, 43 and $56 \mathrm{MW}$ on the left of Fig. 8. Vibrations measured in the same bearing at $90^{\circ}$ show similar results. However, the vibration amplitude levels in the thrust bearing are considerably lower specially in axial direction.

The vibrations have been band pass filtered between 5 and $10 \mathrm{kHz}$. Then, the averaged $P S D$ of the analytic signals has been computed and the results are plotted as a function of output power for position A13 on the right of Fig. 8. A significant frequency peak at $f_{v}$ prevails especially at maximum load. A detailed observation of the modulation spectrum at this load also permits to detect the presence of smaller peaks at $f_{b}$ and its harmonics $2 f_{b}$ and $3 f_{b}$. For lower operating conditions all these modulations do not appear. Again, the measurements on the turbine guide bearing give better results than the ones from the thrust bearing. This seems to confirm that erosive leading edge cavitation occurs mainly when the machine operates at maximum load and that it is well detected in the turbine guide bearing.

\subsection{Francis turbine prototype 1}

The experimental results now presented correspond to a vertical Francis turbine that suffers from severe erosion at the middle of the blade suction side next to the band. This damage is 
associated to unstable inlet leading edge cavitation. The turbine nominal flow rate is $28 \mathrm{~m}^{3} / \mathrm{s}$ and the net head is $51 \mathrm{~m}$. The maximum output power is about $11 \mathrm{MW}$ and the rotating speed is $250 \mathrm{rpm}\left(f_{f}=4.16 \mathrm{~Hz}\right)$. The runner has 15 blades and there are 24 guide vanes.

Two accelerometers have been located on the turbine guide bearing in positions A13 and A14. A third accelerometer has been positioned on a guide vane in axial direction in position AGV17. An acoustic emission sensor has been mounted in radial direction in position AE13. Since previous investigations carried out in similar units by Escaler et al. [28,37] and Vizmanos et al. [33] had shown that maximum cavitation was occurring at around full load, the current measurements have been concentrated at $70 \%, 75 \%, 80 \%, 85 \%, 95 \%$ and $100 \%$ guide vane opening $(G V O)$.

The auto-power spectra of the accelerations in position A13 and of acoustic emissions in position AE13 are plotted in Fig. 9 for $70 \%, 85 \%$ and $100 \%$ GVO. It is clear that the spectrum above $3 \mathrm{kHz}$ increases significantly with machine load and this is observed in all the measured positions. In the low frequency range, no spectra separation can be observed.

In reference to the modulating frequencies, Fig. 10 shows the averaged auto-power spectra of demodulated signals in the band from 10 to $15 \mathrm{kHz}$ for the accelerometer on $\mathrm{A} 13$ at $70 \%$ and $100 \%$ GVO. The spectrum at $70 \%$ has a very low amplitude indicating that there is no signal modulation. But, as the load increases some peaks appear and grow. For instance, $f_{b}$ rises significantly at $85 \%$ and becomes the highest peak. At $100 \%$ it is remarkable how $f_{v}$, that has come out at $95 \%$, has the highest amplitude and how $f_{b}$ is not so high. Besides, the $2 f_{v}$ peak also appears and the $f_{f}$ peak and many of its harmonics dominate the low frequency band. In all the cases, analogous qualitative results are obtained with the acoustic emission sensor at higher frequency bands.

Summing up, the modulation spectra at high loads show simultaneously the $f_{v}, f_{b}, f_{f}$ and some harmonics. Again, the amplitude of the modulating frequencies is very sensitive to the operation condition. In fact, the strongest modulation in terms of amplitude and number of frequencies is found at full load in all the sensors including the guide vane. However, it must be noted that there is a poor similitude between results from A13 and AGV17. For instance at full load, in the guide vane the $f_{b}$ peak is one of the lowest ones but $2 f_{b}$ and $3 f_{b}$ are the highest ones, which does not happen in $\mathrm{A} 13$.
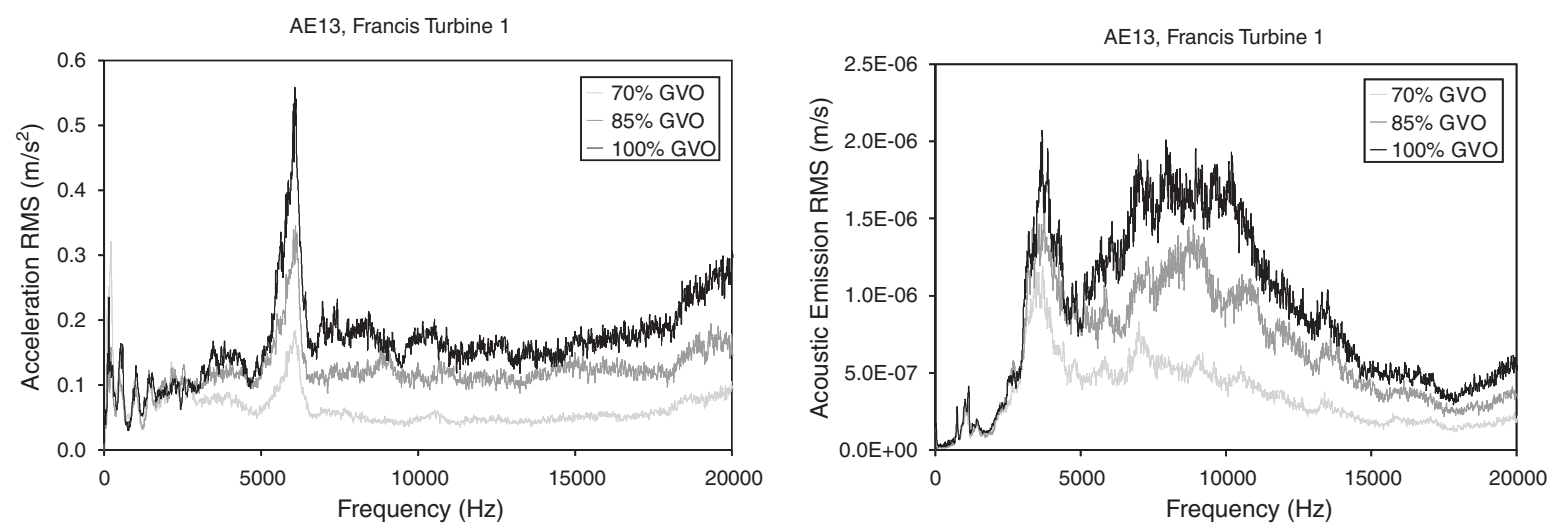

Fig. 9. Auto-power spectra of vibrations in A13 and of acoustic emissions in AE13 at different GVO's for the Francis turbine prototype 1 . 

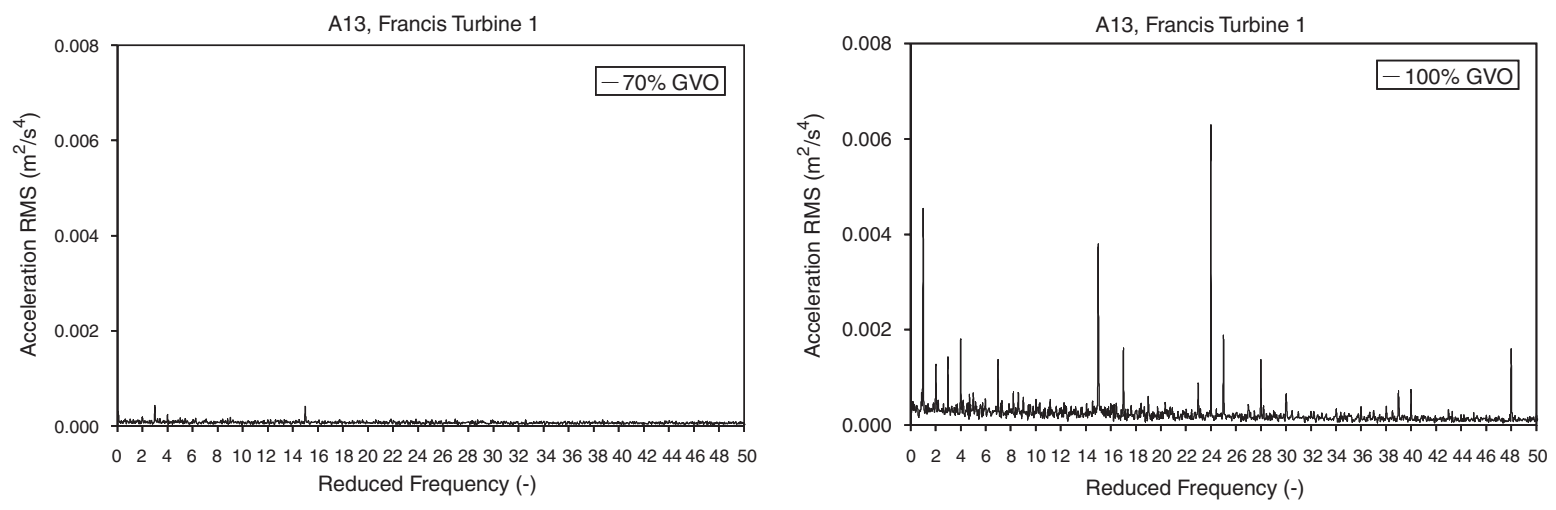

Fig. 10. Modulation auto-power spectra in the band from 10 to $15 \mathrm{kHz}$ of vibrations in $\mathrm{A} 13$ at $70 \%$ and $100 \%$ GVO for the Francis turbine prototype 1 .

\section{Detection of bubble cavitation}

\subsection{Francis turbine model}

A reduced scale model of a Francis turbine prototype that is suited to reproduce bubble cavitation has been used. The experiments have been carried out in the test rig of the Laboratory for Hydraulic Machinery of the Swiss Federal Institute of Technology. In this testing facility, the maximum flow rate can be set to $1.4 \mathrm{~m}^{3} / \mathrm{s}$ and the maximum net head to 1 $00 \mathrm{~m}$. For the current tests, the rotating speed has been fixed to $874 \mathrm{rpm}\left(f_{f}=14.6 \mathrm{~Hz}\right)$. The turbine has 19 blades and 20 guide vanes. A typical example of erosion occurred at the outlet of the blades of a Francis turbine prototype due to bubble cavitation is shown on the left of Fig. 11.

The model has been functioned in two operating conditions keeping the design head constant. For the first condition, the flow rate has been kept at its nominal value with no cavitation at all in the machine, neither inside the runner nor in the draft tube. For the second, the flow rate has been extremely increased until severe travelling bubble cavitation has been visually observed through the transparent draft tube wall with the help of a stroboscopic lamp. In this case, a strong swirl has also appeared.

One accelerometer has been mounted radially in position A13, and a second one axially at $180^{\circ}$ in position A15, both in the turbine bearing. Furthermore, two dynamic-pressure sensors have been flush-mounted on the draft tube wall in positions PDT13 and PDT11.

The auto-power spectra of vibrations in position A13 are plotted on the right of Fig. 11. The high frequency content of the signal is always higher when cavitation occurs. The amplitude of the measured vibrations indicates that the machine is severely excited as expected from the visual observations. Similar results are obtained in axial direction in position A15, but the vibration amplitude is lower than in radial direction. Besides, the shape of the spectra differs between radial and axial directions. For the pressure signals in the draft tube wall, it is also observed that the amplitudes increase with the presence of cavitation. 

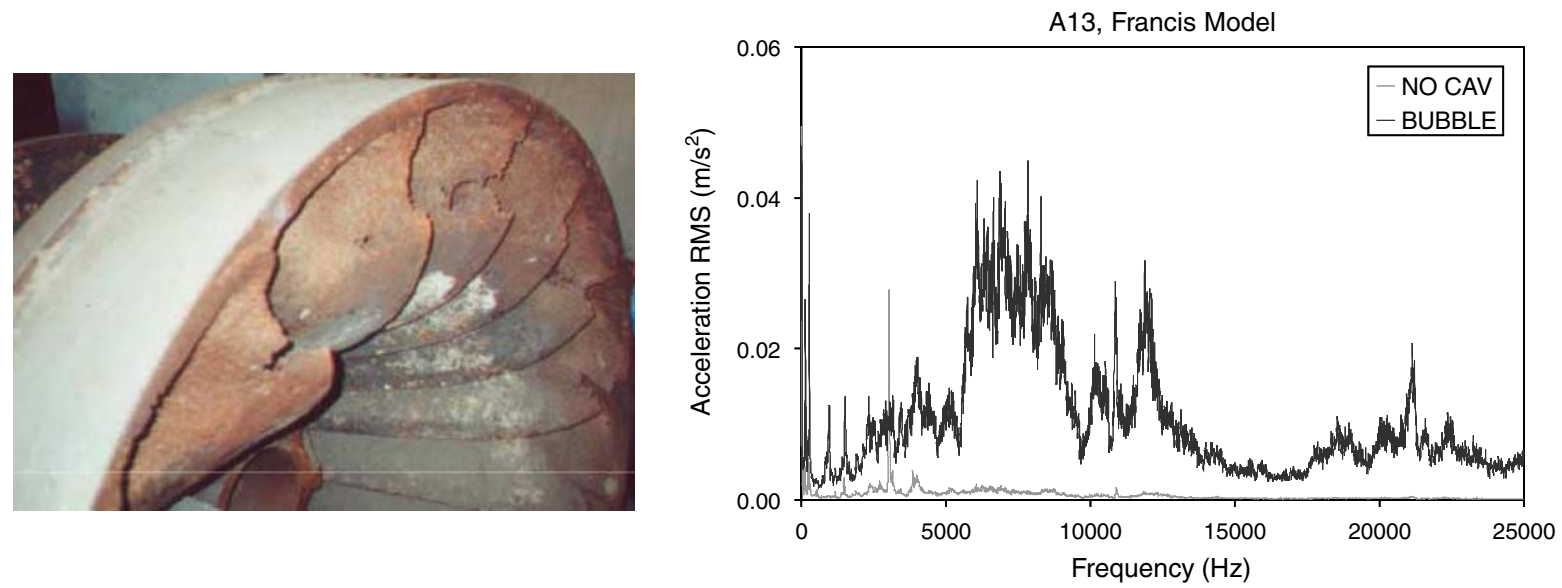

Fig. 11. (Left) Photograph of a prototype runner with advanced outlet erosion on the blades due to bubble cavitation. (Right) Comparison of auto-power spectra of vibrations in A13 without cavitation and with bubble cavitation for the Francis turbine model.
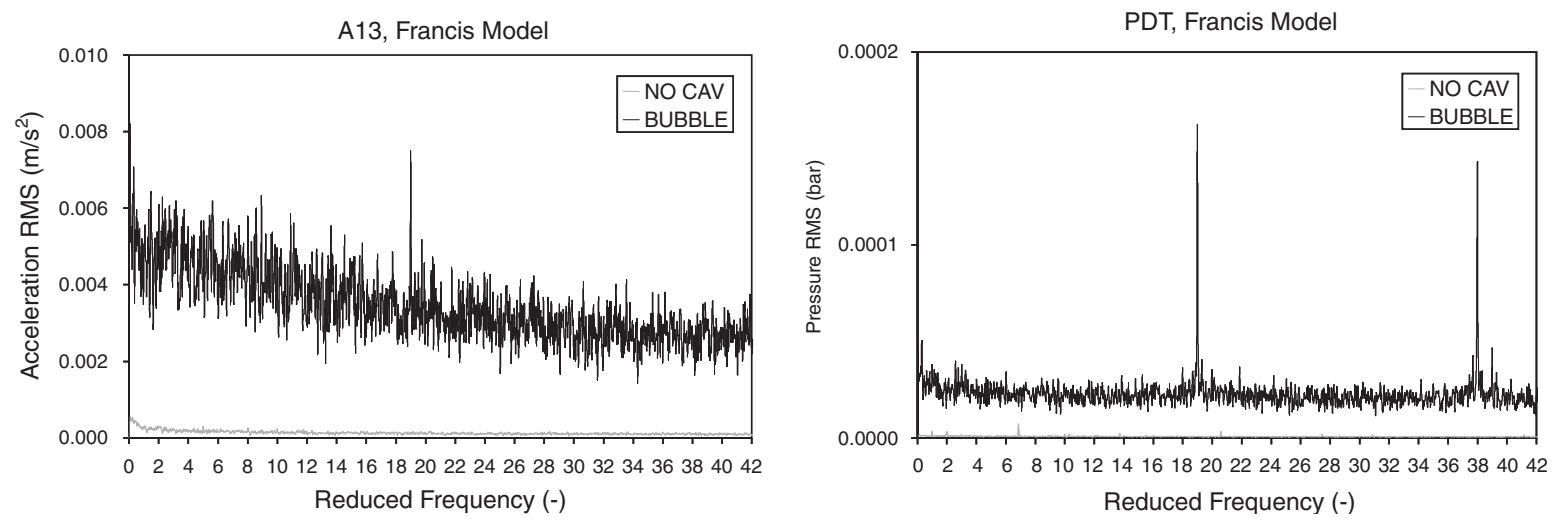

Fig. 12. Comparison of modulation auto-power spectra in the band from 10 to $15 \mathrm{kHz}$ with no cavitation and with bubble cavitation of vibrations in A13 (left) and of pressures in PDT (right) for the Francis turbine model.

The amplitude demodulation of vibrations in position A13 and of dynamic pressures in position PDT13 filtered in the frequency band from 10 to $15 \mathrm{kHz}$ are shown in Fig. 12. In the situation without cavitation no notable hydrodynamic frequency appears on the auto-power spectrum of the envelope. When bubble cavitation is observed, mainly $f_{b}$ predominates in the vibration modulation spectrum and both $f_{b}$ and $2 f_{b}$ predominate in the pressure modulation spectrum. For the vibrations, the results are more clear in radial than in axial direction. For the pressures, both sensors at $180^{\circ}$ show similar results. So, in this case, a distinctive signature is detected when applying the amplitude demodulating technique to bubble cavitation from both structure-borne and from fluid-borne noise. 


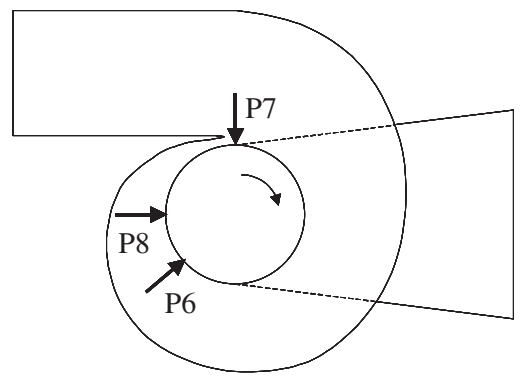

P7-P8, Pump-Turbine

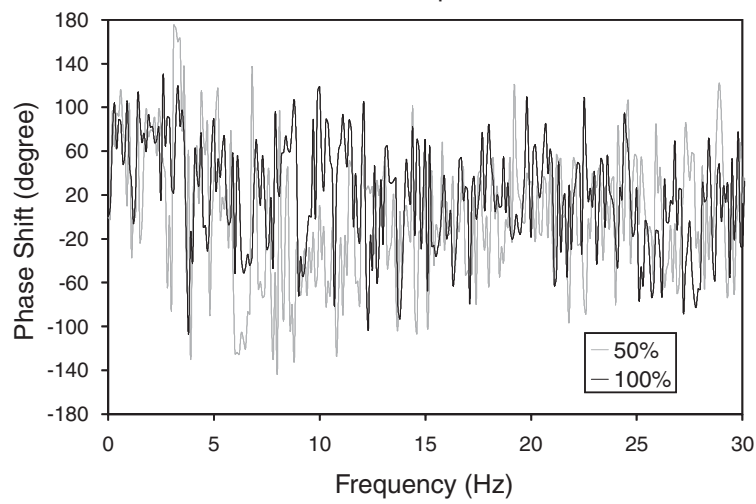

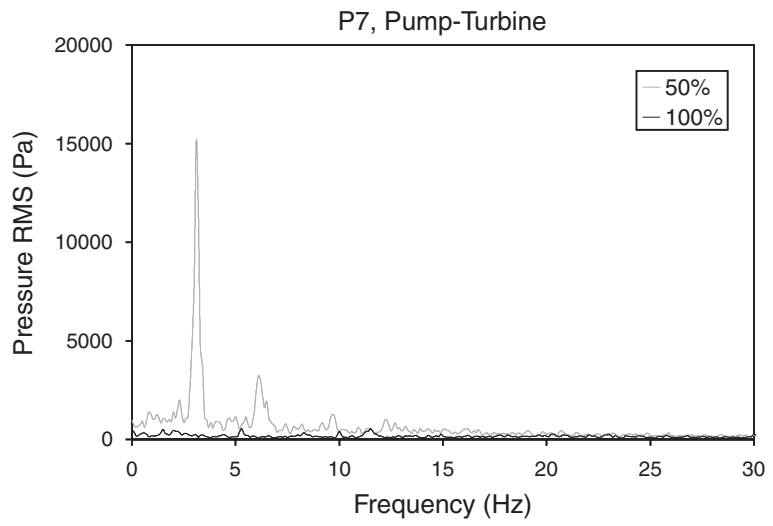

P7-P8, Pump-Turbine

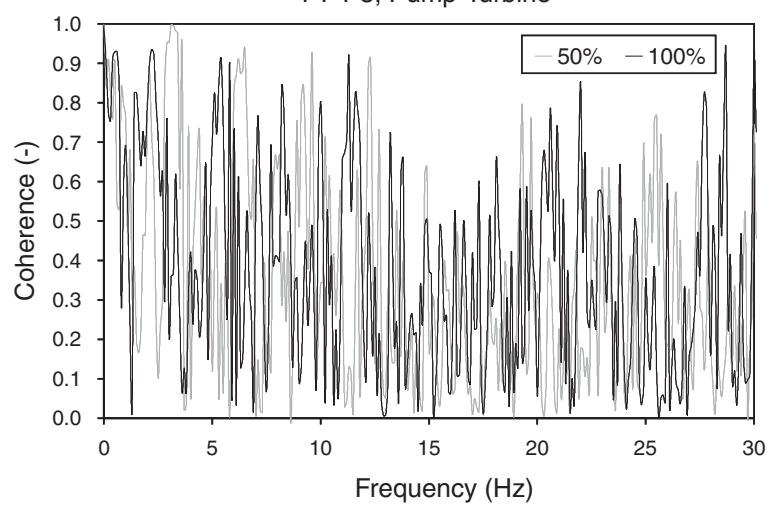

Fig. 13. (Top left) Location of the pressure transducers in the draft tube of the Pump-Turbine prototype. (Top right) Auto-power spectra of pressure P7 at 50\% and 100\% output load. (Bottom left) Phase shift between pressures P7 and $\mathrm{P} 8$ at $50 \%$ and $100 \%$ output load. (Bottom right) Coherence between pressures P7 and P8 at 50\% and 100\% output load.

\section{Detection of draft tube swirl}

\subsection{Pump-Turbine prototype}

The experimentation in a high-head single-stage reversible Pump-Turbine is presented. This vertical machine has a rotational speed of $600 \mathrm{rpm}\left(f_{f}=10 \mathrm{~Hz}\right)$ and permits a maximum output load of $112.75 \mathrm{MW}$. The total head is $400 \mathrm{~m}$ and the flow rate is $24 \mathrm{~m}^{3} / \mathrm{s}$ in pumping mode and can reach $31.5 \mathrm{~m}^{3} / \mathrm{s}$ in turbine mode. This unit has seven blades and 16 guide vanes.

Three dynamic-pressure transducers have been flush-mounted in the draft tube wall. Two of them, named P7 and P8, have been located at the same height and separated $90^{\circ}$, as indicated in Fig. 13, and the third one has been positioned in a lower plane and separated $45^{\circ}$ from P8. The tests have been conducted during turbine mode operating at $50 \%, 75 \%$ and $100 \%$ of the maximum output power. During the partial load condition at $50 \%$, the pressure signals are dominated by a strong pulsation at $3.1 \mathrm{~Hz}$ that corresponds to 0.31 times the $f_{f}$. Its second 
harmonic also appears. A high coherence level (around 1) is found between all the transducers at this particular frequency, but it is not the case at higher loads as it can be seen at the bottom right of the same Fig. 13. If the phase difference between pressure pulsations at $3.1 \mathrm{~Hz}$ is calculated between the two sensors located at $90^{\circ}, \mathrm{P} 7$ and $\mathrm{P} 8$, a value around $175^{\circ}$ is found. This strong fluctuation disappears at higher flow rates as it is seen on the top right of Fig. 13 where the autopower spectra for P7 are plotted at $50 \%$ and $100 \%$ output load. Thus, these results confirm the detection of a cavitating vortex core with a helical shape in the draft tube at $50 \%$ load that rotates in the same direction as the runner at 0.31 times $f_{f}$.

\subsection{Francis turbine prototype 2}

Apart from the low frequency pressure pulsations, a vortex rope in the draft tube can provoke high frequency noise that can travel up to the shaft (see the right of Fig. 5) and that can be detected from measurements on the bearing. This is the case of a vertical Francis turbine that can operate up to a maximum output power of $65 \mathrm{MW}$. The total nominal flow rate is $115 \mathrm{~m}^{3} / \mathrm{s}$ and the net head is around $122.5 \mathrm{~m}$. The rotating speed is $250 \mathrm{rpm}\left(f_{f}=4.16 \mathrm{~Hz}\right)$, the number of blades is 15 and the number of guide vanes is 24 . In particular, this unit has a limitation in the operating range below $30 \mathrm{MW}$ due to the apparition of a swirl that generates strong vibrations on the draft tube walls.

The turbine guide bearing vibrations have been measured in radial position A12 and the acoustic emissions in axial position AE16. Two more accelerometers have been located on the top of two guide vanes in positions AGV17 and AGV18. The unit has been operated at 20, 25, 30, 35, 40, 45, 50, 55, 60 and $65 \mathrm{MW}$.

The overall root mean square $(R M S)$ values of vibrations and acoustic emissions have been calculated in the band up to $49 \mathrm{kHz}$ and they have been compared as a function of output power in Fig. 14. It is observed that the vibration levels follow similar trends in the turbine guide bearing and in the guide vanes. The maximum values are detected at 30 and $60 \mathrm{MW}$. However, guide vane vibrations are much higher than guide bearing ones. Considering the auto-power spectra, the entire frequency band above several $\mathrm{kHz}$ is increased or decreased as the load is changed. Nevertheless, there are wideband regions in the high frequency range where the change is larger. This is particularly clear for sensors A12 and AE16 at 30,40 and 60 MW in Fig. 15. For instance, A12 presents maximum amplitudes around $40 \mathrm{kHz}$. The high frequency region is very sensitive to the operating condition.

The envelope's frequency content in the band from 30 to $40 \mathrm{kHz}$ reveals well-defined peaks at certain hydrodynamic frequencies, as shown in Fig. 16. For position A12, the vibration is mainly modulated at 0.27 times $f_{f}$ when the machine operates at loads below $30 \mathrm{MW}$. On the contrary, the $f_{v}$ predominates at higher loads above $50 \mathrm{MW}$. The maximum amplitude for the peak at $0.27 f_{f}$ occurs at $30 \mathrm{MW}$ and for $f_{v}$ at $60 \mathrm{MW}$. The AE16 sensor leads exactly to the same results. At $30 \mathrm{MW}$, the base level of the spectrum is highly increased as if strong turbulence were associated to this operating condition. If the guide vane sensor is considered, $f_{b}$, some harmonics and $f_{f}$ are also important. The peak at $0.27 f_{f}$ suggests the presence of a partial load cavitation swirl inside the draft tube. The peak at $f_{v}$ indicates that erosive inlet cavitation occurs on the blades, which is confirmed from inspection. Both frequencies are very well detected in the turbine guide bearing by the accelerometer and the acoustic emission sensor. 

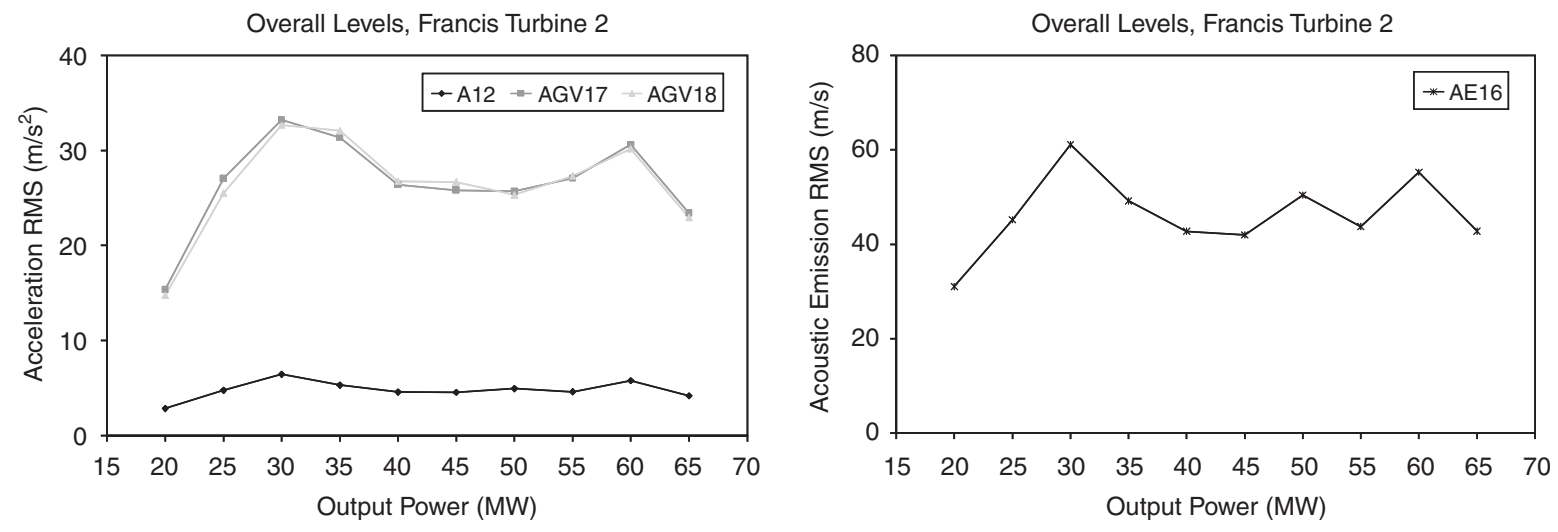

Fig. 14. Overall RMS levels of vibrations in A12, AGV17 and AGV18, and of acoustic emissions in AE16 at various output loads for the Francis turbine prototype 2.
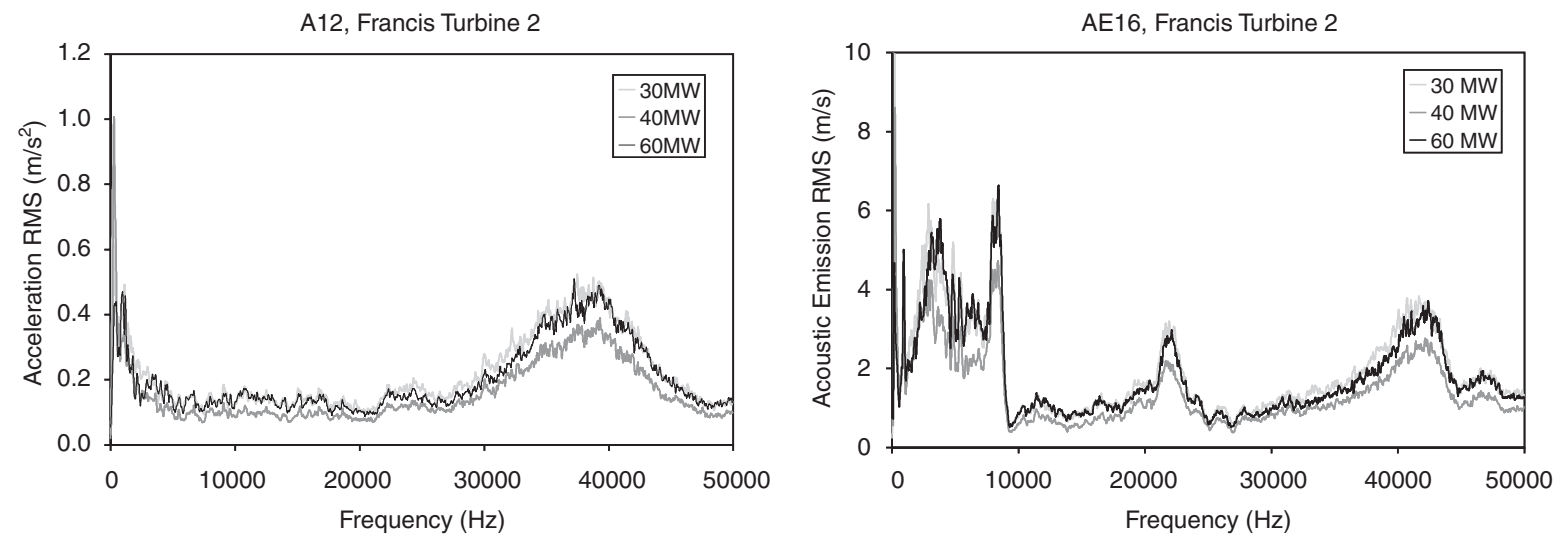

Fig. 15. Auto-power spectra of vibrations in A12 and of acoustic emissions in AE16 at various output loads for the Francis turbine prototype 2 .
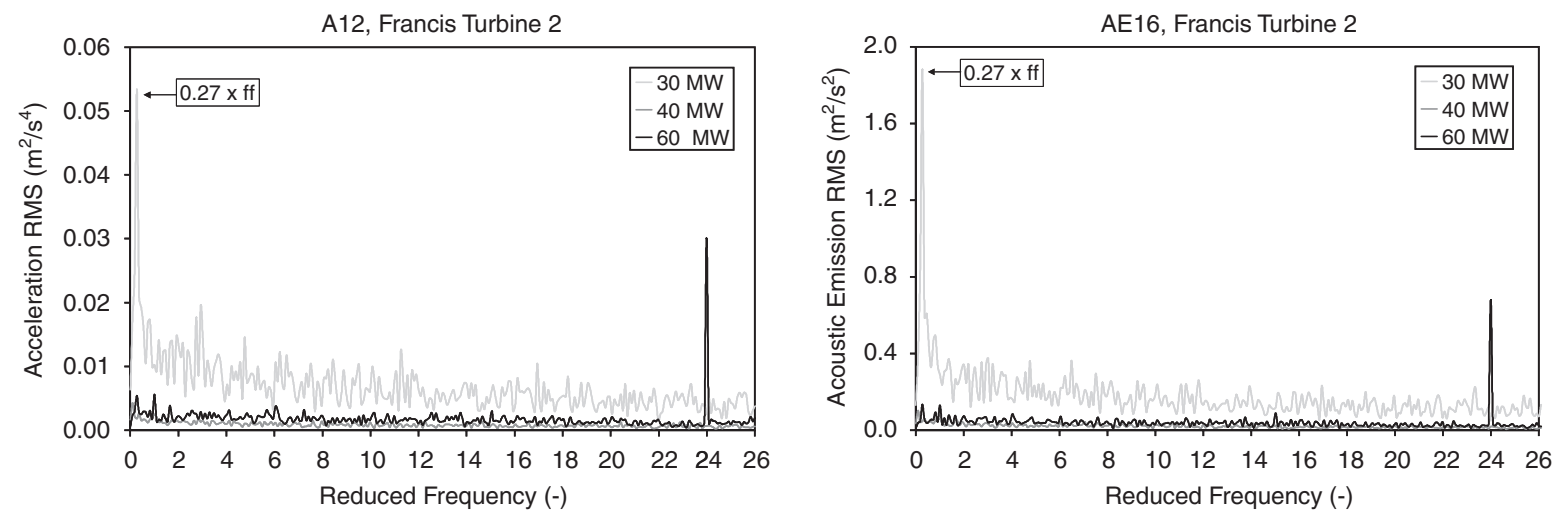

Fig. 16. Modulation auto-power spectra in the band from 30 to $40 \mathrm{kHz}$ of vibrations in A12 and of acoustic emissions in AE16 at various output loads for the Francis turbine prototype 2. 


\section{Conclusions}

Cavitation can present different forms in hydraulic turbines depending on the machine design and the operating condition. As a result, high vibration levels, instabilities and erosion can occur which invalidates the machine operation and provokes damage.

The types of cavitation of major interest in hydraulic turbines are the inlet leading edge cavitation, the outlet bubble cavitation and the draft tube swirl, which have been described after introducing the basic cavitation phenomena.

Various machines suffering from these types of cavitation have been selected for the experimental investigation. In particular one Kaplan, two Francis and one Pump-Turbine prototypes with output powers ranging from 11 to $112 \mathrm{MW}$ have been investigated in the field. Additionally, one Francis model located in a laboratory has also been tested.

Some cavitation detection techniques have been proposed based on the previous understanding of the cavity dynamics, that generates the signals, and its location inside the machine, that determines its propagation. This knowledge has been gained from flow visualisations and measurements in laboratory devices such as a high-speed cavitation tunnel and a reduced scale turbine test rig.

Since cavitation induces structural vibrations, acoustic emissions and hydrodynamic pressures, the detection techniques rely on the analysis of these signals. The collapsing process of the individual cavities, that generates signals of high frequency content, is generally governed by a low frequency fluctuation associated to the main cavity dynamics. As a result, the high frequency signals are modulated in amplitude at this low frequency. Moreover, such fluctuation can also generate low frequency signals.

Unstable leading edge cavitation produces vibrations on the runner blades that propagate through the mechanical system. For its detection it is recommended to measure vibrations as close as possible to the runner in the turbine guide bearing pedestal. Radial vibrations are preferred to axial ones. The guide vanes also serve for detection but the results are not so clear. The draft tube wall is not a good detection location. The main hydrodynamic frequencies modulating the vibration amplitude of the signals in a high frequency band of about $5 \mathrm{kHz}$ have to be found. This band should be selected from the previous observation of the raw vibration spectra since its amplitude and shape can change depending on the measuring position and the machine being investigated. A frequency band centred around $10 \mathrm{kHz}$ is usually appropriate for any type of machine measuring in the guide bearing. In this case, a strong modulation at the guide vane passing frequency predominates both in Kaplan and Francis turbines. Nevertheless, an additional modulation peak at the blade passing frequency can also be found in some Francis machines. The amplitude of these frequency peaks is related to the intensity of the cavitation.

For outlet bubble cavitation on the runner blades, the main modulation only seems to occur at the blade passing frequency in a Francis turbine. For its detection, radial vibrations should also be measured in the turbine guide bearing. In this case, pressures in the draft tube wall are also useful but guide vane vibrations are not recommended.

In fact, for all types of cavitation there is an increase of the high frequency vibration levels above $3 \mathrm{kHz}$. As the cavitation severity grows, the amplitude of the entire spectrum increases without changing its shape significantly. Results obtained with acoustic emission sensors at much 
higher frequencies are analogous to results obtained with accelerometers in lower frequency bands.

For detection of draft tube swirl, an adequate technique is the use of draft tube wall pressure measurements to identify the pulsation directly from a low frequency spectrum at around 0.3 times $f_{f}$. In a large Francis turbine, a modulation frequency at approximately the same value can also be found in the high frequency vibrations measured in the guide bearing.

In summary, the most adequate sensor, measuring position and signal processing have been demonstrated for each type of cavitation.

\section{Acknowledgements}

ENDESA GENERACIÓN and the CICYT (DPI2000-0702) are thanked for their financial support and collaboration. Our gratitude is also conveyed to the staff of CDIF and LMH-EPFL for their technical support.

\section{Appendix. Nomenclature}

$\begin{array}{ll}C & \text { absolute velocity }(\mathrm{m} / \mathrm{s}) \\ C_{I} & \text { suction section mean velocity }(\mathrm{m} / \mathrm{s}) \\ E & \text { specific energy }(\mathrm{Nm} / \mathrm{Kg}) \\ f & \text { frequency }(\mathrm{Hz}) \\ f_{b} & \text { blade passing frequency }(\mathrm{Hz}) f_{b}=Z_{b} f_{f} \\ f_{f} & \text { fundamental frequency }(\mathrm{Hz}) f_{f}=N / 60 \\ f_{v} & \text { guide vane passing frequency }(\mathrm{Hz}) f_{v}=Z_{v} f_{f} \\ f^{*} & \text { reduced frequency }(-) f^{* *}=f / f_{f} \\ g & \left.\text { gravity (m/s }{ }^{2}\right) \\ H i & \text { Hilbert transform }(-) \\ H_{s} & \text { machine setting level (m) } \\ N & \text { rotating speed (rpm) } \\ p_{a} & \text { downstream-free surface pressure }(\mathrm{Pa}) \\ p_{B} & \text { bubble pressure }(\mathrm{Pa}) \\ p_{I} & \text { suction section pressure }(\mathrm{Pa}) \\ p_{v} & \text { vapour pressure }(\mathrm{Pa}) \\ p_{\infty} & \text { infinite domain pressure }(\mathrm{Pa}) \\ Q & \text { flow rate }\left(\mathrm{m}^{3} / \mathrm{s}\right) \\ r & \text { radius }(\mathrm{m}) \\ R & \text { runner external radius }(\mathrm{m}) \\ R_{B} & \text { bubble radius }(\mathrm{m}) \\ t & \text { time }(\mathrm{s}) \\ U & \text { tangential velocity }(\mathrm{m} / \mathrm{s}) U=\omega r \\ W & \text { relative fluid velocity }(\mathrm{m} / \mathrm{s}) \\ x(t) & \text { time signal }(\mathrm{s})\end{array}$




$\begin{array}{ll}Z_{b} & \text { number of runner blades }(-) \\ Z_{I} & \text { suction section height }(\mathrm{m}) \\ Z_{\text {ref }} & \text { reference section height }(\mathrm{m}) \\ Z_{v} & \text { number of guide vanes }(-) \\ \alpha & \text { incidence angle (deg.) } \\ \beta & \text { guide vane angle (deg.) } \\ \gamma & \text { surface tension }(\mathrm{N} / \mathrm{m}) \\ \eta & \text { efficiency }(-) \\ \varphi & \text { flow coefficient }(-) \\ v & \text { kinematic viscosity }\left(\mathrm{m}^{2} / \mathrm{s}\right) \\ \rho & \text { density }\left(\mathrm{Kg} / \mathrm{m}^{3}\right) \\ \sigma_{p} & \text { Thoma coefficient }(-) \\ \tau & \text { Rayleigh time }(\mathrm{s}) \\ \omega & \text { angular velocity }(\mathrm{rad} / \mathrm{s}) \\ \psi & \text { pressure coefficient }(-) \\ G V O & \text { guide vane opening }(\%) \\ N P S E & \text { net positive suction energy }(\mathrm{Nm} / \mathrm{Kg}) \\ P S D & \text { power spectral density }\left(\mathrm{m}^{2} / \mathrm{s}^{4} / \mathrm{Hz}\right) \\ R M S & \text { root mean square }(-)\end{array}$

\section{References}

[1] O. Coutier-Delgosha, R. Fortes-Patella, J.L. Reboud, M. Hofmann, B. Stoffel, Experimental and numerical studies in a centrifugal pump with two-dimensional curved blades in cavitating condition, Journal of Fluids Engineering 125 (2003) 970-978.

[2] M. Cudina, Detection of cavitation phenomenon in a centrifugal pump using audible sound, Mechanical Systems and Signal Processing 17 (6) (2003) 1335-1347.

[3] A. Baldassarre, M. De Lucia, P. Nesi, Real-time detection of cavitation for hydraulic turbomachines, Real-Time Imaging 4 (1998) 403-416.

[4] P.J. McNulty, I.S. Pearsall, Cavitation inception in pumps, Journal of Fluids Engineering 104 (1982) 99-104.

[5] F. Avellan, P. Henry, Towards the prediction of cavitation erosion: IMHEF research program, Proceedings of the EPRY Symposium on Power Plant Pump, New Orleans, USA, 1987, pp. 1-22.

[6] P. Bourdon, R. Simoneau, P. Lavigne, A vibratory approach to the detection of erosive cavitation, Proceedings of the Third International Symposium on Cavitation Noise and Erosion in Fluid Systems, FED-vol. 88, ASME Winter Annual Meeting, San Francisco, CA, 1989, pp. 103-109.

[7] R.T. Knapp, J.W. Daily, F.G. Hammit, Cavitation, McGraw-Hill, New York, 1970.

[8] F. Avellan, M. Farhat, Shock pressure generated by cavitation vortex collapse, Proceedings of the Third International Symposium on Cavitation Noise and Erosion in Fluid Systems, FED-vol. 88, ASME Winter Annual Meeting, San Francisco, CA, 1989, pp. 119-125.

[9] A. Philipp, W. Lauterborn, Cavitation erosion by single laser-produced bubbles, Journal of Fluid Mechanics 361 (1998) 75-116.

[10] F.G. Hammit, Cavitation erosion: the state of the art and predicting capability, Applied Mechanics Reviews 32 (6) (1979) 665-675.

[11] R.E.A. Arndt, Recent advances in cavitation research, in: Advances in Hydroscience, vol. 12, Academic Press, New York, 1981, pp. 1-72.

[12] C.E. Brennen, Cavitation and Bubble Dynamics, Oxford University Press, Oxford, 1995. 
[13] F. Avellan, Ph. Dupont, I.L. Ryhming, Generation mechanism and dynamics of cavitation vortices downstream of a fixed leading edge cavity, Proceedings of the 17th Symposium on Naval Hydrodynamics, vol. V, The Hague, Netherlands, 1988, pp. 1-13.

[14] J.P. Franc, F. Avellan, B. Belahadji, J.Y. Billard, L. Briançon-Marjollet, D. Fréchou, D.H. Fruman, A. Karimi, J.L. Kueny, J.M. Michel, La Cavitation. Mécanismes Physiques et Aspects Industriels, Presses Universitaires de Grenoble, 1995.

[15] W.P. Creager, J.D. Justin, Hydroelectric Handbook, Wiley, New York, 1950.

[16] J. Raabe, Hydro Power: the Design, Use and Function of Hydromechanical, Hydraulic and Electrical Equipment, VDI-Verlag GMBH, Dusseldorf, 1985.

[17] L. Vivier, Turbines Hydrauliques et leur Régulation: Théorie, Construction et Utilisation, Albin Michel, Paris, 1966.

[18] S.C. Li (Ed.), Cavitation of Hydraulic Machinery, vol. 1, Imperial College Press, London, 2000.

[19] B. Grindoz, Lois de Similitudes dans les Essays de Cavitation des Turbines Francis, Ph.D. Thesis 714, EPFL, Lausanne, 1991.

[20] G.J. Causon, Sonic cavitation studies on model and prototype water turbines, Mechanical \& Chemical Engineering Transactions, Institution of Engineers, Australia Mechanical and Chemical Engineering Transactions, Australia MC8 (1) (1972) 24-30.

[21] K. Steller, J. Kirejczyk, Diagnostic of cavitation in the hydraulic machinery, The Transaction of the Institute of Fluid-Flow Machinery, Gdansk, no. 86, 1983, pp. 3-39.

[22] P.A. Abbot, C.J. Gedney, D.L. Greeley, Cavitation monitoring of two axial-flow hydroturbines using novel acoustic and vibration methods, Proceedings of the 13th IAHR Symposium, Montreal, Canada, vol. 1, Paper 23, 1986.

[23] B. Bajic, A. Keller, Spectrum normalization method in vibro-acoustical diagnostic measurements of hydroturbine cavitation, Journal of Fluids Engineering 118 (1996) 756-761.

[24] P. Bourdon, M. Farhat, R. Simoneau, F. Pereira, Ph. Dupont, F. Avellan, J.M. Dorey, Cavitation erosion prediction on francis turbines. Part 1: measurements on the prototype, Proceedings of the 18th IAHR Symposium on Hydraulic Machinery and Cavitation, Valencia, Spain, vol. 1, 1996, pp. 534-543.

[25] H.J. Baiter, On different notions of cavitation noise and what they imply, Proceedings of the International Symposium on Cavitation and Multiphase Flow, FED-vol. 45, ASME, 1986, pp. 107-118.

[26] S.L. Ceccio, C.E. Brennen, Observations of the dynamics and acoustics of travelling bubble cavitation, Journal of Fluid Mechanics 233 (1991) 633-660.

[27] P.A. Abbot, Cavitation detection measurements on Francis and Kaplan hydro turbines, Proceedings of the Third International Symposium on Cavitation, Noise and Erosion in Fluid Systems, FED-vol. 88, ASME Winter Annual Meeting, San Francisco, CA, 1989, pp. 55-61.

[28] X. Escaler, E. Egusquiza, T. Mebarki, F. Avellan, M. Farhat, Cavitation detection and erosion prediction in hydro turbines, Proceedings of the Ninth of International Symposium on Transport Phenomena and Dynamics of Rotating Machinery, Honolulu, Hawaii, 2002.

[29] M. Farhat, F. Avellan, F. Pereira, Pressions instationnaires generees par une poche de cavitation partielle, La Houille Blanche 7/8 (1992) 579-585.

[30] P.A. Abbot, R.E.A. Arndt, T.B. Shanahan, Modulation noise analyses of cavitating hydrofoils, in: Bubble Noise and Cavitation Erosion in Fluid Systems, FED-vol. 176, ASME, 1993, pp. 83-94.

[31] M. Callenaere, J.P. Franc, J.M. Michel, M. Riondet, The cavitation instability induced by the development of a reentrant jet, Journal of Fluid Mechanics, 2001.

[32] M. Kaye, M. Farhat, Classification of cavitation in hydraulic machines using vibration analysis, Proceedings of the XXIst IAHR Symposium on Hydraulic Machinery and Systems, Lausanne, Switzerland, 2002.

[33] C. Vizmanos, E. Egusquiza, E. Jou, Cavitation detection in a Francis turbine, Conference Monitoring for Hydro Powerplants II, Lausanne, Switzerland, 1996.

[34] P. Bourdon, R. Simoneau, F. Avellan, Erosion vibratory fingerprint of leading edge cavitation of a Naca profile and of a Francis model and prototype hydroturbine, ASME Bubble Noise and Cavitation Erosion in Fluid Systems, FED-vol. 176, 1993. 
[35] M. Farhat, P. Bourdon, P. Lavigne, Some hydro Quebec experiences on the vibratory approach for cavitation monitoring, Conference Monitoring for Hydro Powerplants II, Lausanne, Switzerland, 1996.

[36] M. Farhat, P. Bourdon, P. Lavigne, R. Simoneau, The hydrodynamic aggressiveness of cavitating flows in hydro turbines, ASME Fluids Engineering Division Summer Meeting, FEDSM 9, 1997.

[37] X. Escaler, E. Egusquiza, T. Mebarki, M. Farhat, F. Avellan, Field assessment of cavitation detection methods in hydropower plants, Proceedings of the XXIst IAHR Symposium on Hydraulic Machinery and Systems, Lausanne, Switzerland, 2002. 